\title{
Temperatura neutral y rangos de confort térmico para exteriores, período cálido en clima cálido seco
}

\author{
Neutral temperature and thermal comfort ranges for \\ outdoors, warm period in warm dry climate
}

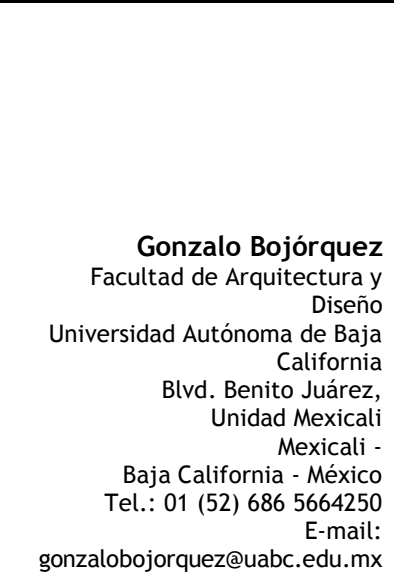

Luis Gabriel GómezAzpeitia

Facultad de Arquitectura y Diseño

Universidad de Colima Campus Coquimatlán Coquimatlán - Colima - México

Tel.: 01 (52) 3123161161

E-mail: ggomez@ucol.mx

Onofre Rafael GarcíaCueto

Instituto de Ingeniería Universidad Autónoma de Baja California

Tel.: 01 (52) 6865664150 E-mail:

rafaelcueto@uabc.edu.mx

Raúl Pavel Ruiz-Torres Facultad de Arquitectura Universidad Autónoma de Chiapas

Blvd. Belisario Domínguez, km 1081

Tuxtla Gutierrez - Chiapas México

Tel.: 01 (52) 9616150935

E-mail: pavelvvg@msn.com

Aníbal Luna

Facultad de Arquitectura y Diseño

Universidad Autónoma de Baja California

Tel.:01 (52) 6865664250 E-mail: anibal@uabc.edu.mx

Recebido em 06/03/2010 Aceito em 26/04/2010

\section{Gonzalo Bojórquez \\ Luis Gabriel Gómez-Azpeitia \\ Onofre Rafael García-Cueto \\ Raúl Pavel Ruiz-Torres \\ Aníbal Luna}

\section{Resumen}

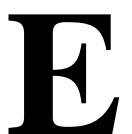

l estimar las temperaturas de confort térmico en espacios exteriores permite obtener información que sirve de base para generar propuestas de diseño, cuyas condiciones ambientales propicien el confort térmico de los usuarios. Se presenta la estimación de temperatura neutral y rangos de confort térmico para espacios exteriores de un parque recreativo, en el período cálido en un clima desértico. El estudio fue desarrollado en Mexicali, Baja California, México. Se diseñó un cuestionario basado en la escala de sensaciones térmicas de ISO 10551, se midieron temperatura de bulbo seco, temperatura de globo gris, humedad relativa y velocidad de viento. Se aplicaron 822 encuestas en julio y agosto del 2008. Se estimaron temperatura neutra y rangos de confort con el método de medias por intervalo de sensación térmica. El análisis se hizo para tres niveles de actividad: pasiva, moderada e intensa y uno combinado con los tres niveles. Los valores de temperatura neutra obtenidos son aproximadamente simétricos con respecto a sus rangos de confort térmico. Las temperaturas neutras obtenidas muestran que los sujetos en actividad intensa, con práctica periódica de ejercicio y hábitos apropiados a las condiciones de clima, tienen una temperatura de confort térmico similar a aquellos con actividad pasiva.

Palabras clave: Modelo adaptativo, confort térmico, temperatura neutral, rangos de confort térmico, espacios exteriores.

\section{Abstract}

Estimating thermal comfort temperatures in outdoor spaces provides valuable information that can be used to generate design proposals whose environmental conditions ensures thermal comfort for the users. This study shows the estimated neutral temperature and thermal comfort ranges during the warm period in a public recreational green area located in a desert climate. It was carried out in Mexicali, Baja California, Mexico. A questionnaire was designed based on the scale of thermal sensation of ISO 10551, and measurements of dry bulb temperature, gray globe temperature, relative humidity and wind speed were taken. A total of 822 questionnaires were applied during the months of July and August 2008. The neutral temperature and comfort range were estimated using a statistical method of correlation. The analysis was undertaken for three activity levels, passive, moderate e intense, as well as for the three levels together. The neutral temperature values were approximately symmetrical with respect to the thermal comfort range. They also indicated that intensely active subjects who exercise regularly and have habits that are appropriate to the weather conditions had a comfort temperature similar to that of more passive subjects.

Key words: Adaptive model, thermal comfort, neutral temperature, thermal comfort ranges, outdoor spaces. 


\section{Introducción}

El cuerpo humano es sometido, en general, a diferentes condiciones climáticas, y en particular a cambios de temperatura, humedad, velocidad de viento y radiación solar, que crean la sensación de comodidad o incomodidad en el individuo. El conocer las condiciones de confort térmico en exteriores, establece las bases para una correcta toma de decisiones en el diseño de espacios, lo que puede propiciar que los usuarios estén en ambiente térmico apto para el desarrollo de sus actividades de manera adecuada.

La necesidad de investigación sobre la sensación térmica percibida en exteriores, se ha visto en eventos como juegos olímpicos y ferias mundiales (PICKUP; DE DEAR, 2000), además de proyectos como Rediscovering the Urban Realm and Open Spaces (Nikolopoulou, 2004), las aportaciones de estos trabajos tienen aplicaciones en proyectos de tipo turístico, recreativo ó áreas de exposiciones. Ashihara (1982) define los espacios exteriores como aquellos que se crean al delimitar la naturaleza y generan un entorno exterior con un fin específico. Este tipo de espacios no son cubiertos y están definidos por dos planos: pisos y muros.

El estudio de la relación clima-humano es el origen de la bioclimatología, definida como área de la ciencia, que estudia la interacción entre los seres vivos y el ambiente atmosférico donde se desenvuelven (AULICIEMS, 1998). En bioclimatología existe un interés particular sobre el estudio, estimación y simulación del confort térmico humano, definido en la norma ISO 7730 (2005) como "aquella condición mental que expresa satisfacción con el ambiente térmico"; que también puede definirse según Nikolopoulou (2004) como "la satisfacción psicofisiológica del humano con respecto a las condiciones climáticas del entorno". Según Humpreys y Nicol (1998) para los estudios de confort térmico se utilizan dos enfoques:

(a) enfoque de predicción: Se trabajan datos de laboratorio, con condiciones de prueba controladas. El individuo se estudia aislado de su hábitat y se consideran las reacciones fisiológicas (aclimatación) voluntarias e involuntarias para alcanzar el confort térmico. Se considera al humano como receptor pasivo en espera del balance energético. Los modelos de este enfoque tienen un nivel de análisis fisiológico; y

(b) enfoque de adaptación: Se trabajan datos de campo, las condiciones de prueba tienen variación continua y el individuo se estudia en su hábitat. Se consideran reacciones fisiológicas (aclimatación) y psicológicas (expectativa, experiencia, conducta).
Se considera al humano como receptor activo en busca del confort térmico. Los modelos de este enfoque tienen niveles de análisis fisiológico y psicológico.

El estudio realizado por De Dear, Brager e Cooper (1998), contribuyó a la reconciliación de los dos enfoques al demostrar la existencia de niveles diferentes de adaptación (fisiológico y psicológico), algo que no era reconocido en el pasado. Las personas se adaptan mediante procesos de ajuste en su organismo y modificaciones en su ambiente térmico, bajo el enfoque de adaptación, para reducir incomodidad y la tensión fisiológica (HUMPHREYS; NICOL, 2002).

Según Nikolopoulou y Steemers (2003), la adaptación térmica humana se considera como el decremento gradual de respuesta del organismo a repetidas exposiciones a estímulos que se reciben de un ambiente térmico específico. En el contexto del confort térmico esta descripción considera todos los procesos en los cuales las personas tienden a mejorar sus condiciones entre el ambiente térmico y sus requerimientos higrotérmicos. La adaptación para su estudio se puede dividir en dos categorías: fisiológica y psicológica.

La adaptación fisiológica implica cambios en las respuestas de la termorregulación humana. En el contexto del medio ambiente térmico esto es llamado aclimatación. La adaptación psicológica establece que la manera de percibir el medio ambiente de las personas es diferente de una a otra, y la respuesta humana a un estímulo físico no está directamente relacionada con su magnitud, pero depende de la información que las personas reciban para una situación en particular. Los factores psicológicos son por lo tanto influidos por la percepción térmica del espacio y los cambios que ocurren en éste, con base en las condiciones del espacio, expectativa y experiencia, tiempo de exposición, control de la percepción y estimulación del medio ambiente (NIKOLOPOULOU; STEEMERS, 2003).

La permanencia por uso en espacios exteriores, es menor a la permanencia en interiores, debido a los tiempos en el proceso de adaptación, lo anterior genera que la aplicación de un modelo de confort térmico diseñado para interiores tenga una tendencia a sobrestimar la sensación real del usuario de exteriores. Esta discrepancia es mayor en condiciones de temperaturas bajas que en las de temperaturas altas. En espacios exteriores, el uso de un modelo de predicción por las implicaciones 
de variabilidad de ambientes, condiciones de los espacios exteriores y tiempos de permanencia, no es adecuado, mientras que la aplicación del método de adaptación, debido a que es resultado de una evaluación de campo, se ajusta mejor a las condiciones exteriores (HÖPPE, 2002).

El objetivo de este artículo es presentar la estimación de temperatura neutral y rangos de confort térmico para espacios exteriores de un centro recreativo, en el período cálido, en un clima desértico, para tres niveles de actividad metabólica.

Los resultados obtenidos coinciden con la teoría de los climas asimétricos y su variabilidad con los rangos de confort térmico en período cálido. En términos generales podría decirse que los valores de temperatura neutra son aproximadamente simétricos con respecto a sus rangos de confort térmico extenso y ajustado. Se observó que los sujetos presentaron variabilidad en cuanto a los valores de confort por nivel de actividad ya que ésta no es directamente decreciente conforme aumentó el nivel de metabolismo global.

Los valores obtenidos de temperatura neutra y rangos de confort, demuestran la teoría de adaptación, ya que los sujetos en actividad intensa, con práctica periódica de ejercicio y hábitos apropiados a las condiciones de clima (vestimenta y consumo de líquidos), tienen una temperatura de confort térmico similar a aquellos con actividad pasiva.

\section{Método}

El método fue estructurado en cuatro apartados:

(a) diseño de la investigación;

(b) variables meteorológicas;

(c) estudio correlacional; y

(d) medias por intervalo de sensación térmica.

Cada uno de ellos es descrito a continuación.

\section{Diseño de la investigación}

El diseño de la investigación se basó en la selección del enfoque de estudio, el cual fue determinado en función de los objetivos y las condicionantes de la investigación, por lo que se hizo un comparativo entre los dos enfoques de estudio del confort térmico: el enfoque de adaptación y el de predicción, para ver cuáles de las características de los mismos, eran convenientes para las necesidades del trabajo a desarrollar. Los parámetros analizados fueron: origen de los datos, tipo de hábitat, reacciones a analizar, tipo de receptor de la información y nivel de análisis, todos los requerimientos coincidieron con el enfoque de adaptación.

Cabe mencionar, que en la selección del enfoque de estudio también se consideraron las ventajas y desventajas de cada uno de ellos. Así como la revisión hecha por Höppe (2002), con respecto al hecho de que los tiempos de permanencia en exteriores indican que es más apropiado un enfoque de adaptación, ya que el enfoque de predicción sobreestimaría el efecto percibido. Además se revisaron los trabajos de Givoni et al. (2003), donde se afirma que en exteriores, por la variación climática y la conducta interactiva es más adecuado el enfoque de adaptación. Nikolopoulou (2004) coincide con los autores mencionados y establece que la experiencia, expectativa y conducta reactiva e interactiva hacen necesario el enfoque de adaptación en exteriores.

El enfoque de adaptación en el estudio del confort térmico establece como parte de su aplicación una investigación basada en correlaciones, lo que conlleva a un diseño de la investigación de tipo "no experimental".

Con respecto a la dimensión temporal, la periodicidad del estudio fue decidida con base en el objetivo de la investigación, y cómo los resultados pudieran ser representativos del fenómeno a estudiar, que fue la sensación térmica percibida por el usuario de espacios exteriores de espacios recreativos en clima cálido seco extremoso, en el período cálido. Debido a lo anterior la dimensión temporal fue de tipo transversal.

Se desarrolló un estudio de tipo correlacional, con el enfoque de adaptación, en el período cálido, durante julio y agosto del 2008. Se utilizó un cuestionario basado en ISO 10551 (ISO, 1995) y se midieron temperatura de bulbo seco, temperatura de globo gris, humedad relativa y velocidad de viento. El análisis de datos se llevó a cabo con el método de medias por intervalo de sensación térmica, propuesto por Gómez-Azpeitia et al. (2007).

Los niveles de actividad analizados fueron tres: pasivo $\left(0\right.$ a $\left.75 \mathrm{~W} / \mathrm{m}^{2}\right)$, moderado $\left(76\right.$ a $\left.183 \mathrm{~W} / \mathrm{m}^{2}\right)$ e intenso $\left(183\right.$ a $\left.600 \mathrm{~W} / \mathrm{m}^{2}\right)$. La relación metabólica de consumo energético se estableció con base en Fanger (1986) y Mondelo et al. (2001). Las actividades de tipo pasivo fueron: caminar, observar, platicar y jugar con niños de forma tranquila. Las actividades de tipo moderada fueron: trotar, ejercicio ligero y jugar con niños de forma interactiva, mientras que las actividades intensas fueron: correr, hacer ejercicio intenso, jugar futbol, basquetbol y beisbol además de acrobacias con bicicleta, patineta y a pie. Además 
se incluyó un análisis con el total de las observaciones realizadas con los diferentes niveles de actividad.

El caso de estudio fue el Centro Recreativo Juventud 2000, donde se practican deportes, ejercicios al aire libre y otras actividades de convivencia. El estudio se realizó en Mexicali, Baja California, ciudad del noroeste de México, que se encuentra a una latitud de $32^{\circ} 39^{\prime} 54^{\prime \prime} \mathrm{N}$ y longitud de $115^{\circ} 27^{\prime} 21^{\prime \prime} \mathrm{O}$, con una altura sobre el nivel del mar de cuatro metros. El clima es de tipo cálido seco extremoso, con temperaturas promedio de máximas de $42{ }^{\circ} \mathrm{C}$ y temperaturas promedio de mínimas de $8^{\circ} \mathrm{C}$ (LUNA et al., 2008).

\section{Variables meteorológicas}

La selección de variables meteorológicas a medir se basó en el efecto de las mismas en la sensación térmica percibida, así como en el análisis de casos de estudio sobre confort térmico en interiores y exteriores. Para elegir los instrumentos de trabajo, se consideraron precisión y rangos de los mismos, la disponibilidad y accesibilidad, además de la complejidad de operación.

En el caso de las variables meteorológicas se revisaron los proyectos: Rediscovering the Urban Realm and Open Spaces (RUROS) (NIKOLOPOULOU, 2004) y RP 884 de ASHRAE (DE DEAR, BRAGER; COOPER, 1998) así como las normas ISO 7730 (ISO, 2005) e ISO 7726 (ISO, 1998), además de los trabajos de Nikolopoulou y Steemers (2003), PickUp y De Dear (2000) y Potter y de Dear (2000).

El proyecto RUROS consideró en su desarrollo la medición de temperatura de bulbo seco, humedad relativa, velocidad de viento, radiación solar y temperatura de globo gris. Con respecto a esta última la propuesta inicial era medir temperatura de globo negro pero ante la expectativa de que no todos los sujetos a estudiar vestían de color oscuro, se consideró el uso de un globo gris, como una tonalidad clara de arropamiento, lo anterior fue sugerido por Humpreys (NIKOLOPOULOU; STEEMERS, 2003).

Los estudios del proyecto RP 884 de ASHRAE, tuvieron distintas variables como parámetros de estudio, pero en todos los casos estuvo presente la temperatura de bulbo seco y la humedad relativa, en algunos otros velocidad de viento y en pocos casos radiación (DE DEAR, BRAGER; COOPER, 1998). Los trabajos de PickUp, De Dear y Potter sobre confort térmico en exteriores consideraron la medición de temperatura del aire, humedad relativa y velocidad de viento, además destaca su análisis de radiación solar y radiación infrarroja
(PICKUP; DE DEAR, 2000; POTTER; DE DEAR, 2000).

En lo que respecta a la precisión y rango, en temperaturas era necesario tener errores menores a $1{ }^{\circ} \mathrm{C}$, por el efecto que esta variación puede ocasionar en la sensación térmica percibida del usuario. Por otro lado, según Luna et al. (2008) en Mexicali B.C. se tienen registros de temperatura máxima extremas de $45^{\circ} \mathrm{C}$ y un record histórico de $54{ }^{\circ} \mathrm{C}$, así como una mínima extrema de $-3{ }^{\circ} \mathrm{C}$, por lo que se estableció un rango en los sensores de temperatura de 1 a $60{ }^{\circ} \mathrm{C}$. En el caso de la humedad relativa existen valores promedios de máximas entre $60 \%$ y $65 \%$ con extremos hasta del $100 \%$. Se consideraron rangos de registro entre el 5 y $100 \%$ debido a los valores de máximos y mínimos extremos registrados.

Con relación a la disponibilidad y accesibilidad de instrumentación, la necesidad reducir el tiempo en desarrollo de tecnología apropiada a las consideraciones técnicas del proyecto, llevo en primera instancia a buscar equipo de tipo comercial con las características necesarias para su uso. Sin embargo, se consideró la posibilidad del desarrollo de alguno de los instrumentos de medición, siempre y cuando esto redujera los costos de adquisición y no requiriera de mucho tiempo en su desarrollo y evaluación. La "accesibilidad" se definió como los costos y tiempos de entrega del equipo.

En el caso de la complejidad de operación, se consideró que los instrumentos a utilizar fueran sencillos, sin requerir de una formación o capacitación de uso amplia. Lo anterior debido a que el estudio se basa en la aplicación de encuestas en espacios exteriores y para lo cual se requirió de un número considerable de personal de apoyo, los cuales fueron alumnos de la Facultad de Arquitectura y Diseño de la Universidad Autónoma de Baja California. Una de las principales características del equipo a utilizar fue que tuviera despliegue en pantalla de la información registrada para así poder anotar en las encuestas las condiciones meteorológicas en las que se desarrolló su aplicación.

Con base en lo anterior, las variables seleccionadas fueron: temperatura de bulbo seco, humedad relativa, velocidad de viento y temperatura de globo gris. Se utilizó un monitor de estrés térmico que registra las variables mencionadas e incluye la velocidad de viento con un anemómetro omnidireccional.

Además se utilizó un sensor de temperatura de globo gris, que fue fabricado a partir de un registrador de datos, con sensor de temperatura. Se usó una pelota de plástico con un diámetro de 
$0,045 \mathrm{~m}$, a la que se le introdujo hasta su centro geométrico el sensor de temperatura y se selló el orificio con silicón, posteriormente se pintó de color gris con una emisividad de 0,5. Lo anterior basado en el reporte de Nikolpoulou y Steemers (2003). El proceso y el equipo cumplen con la mayoría de lo requerido en la norma ISO 7726 (ISO, 1998), por lo que los datos generados son de Clase II.

Debido al efecto sobre la sensación térmica percibida de la complexión física de cada persona, fueron necesarios la utilización de una báscula mecánica, para medir el peso y un flexómetro, para medir altura.

\section{Estudio correlacional}

Para el estudio correlacional se diseñó un cuestionario, con base en la norma ISO 10551 (ISO, 1995) y en el análisis de tres cuestionarios para estudios de confort térmico (GÓMEZAZPEITIA et al., 2007; NIKOLOPOULOU, 2004). Se elaboró un cuestionario preliminar, se desarrolló un manual de aplicación de encuesta y uso del equipo. Se hizo una prueba piloto y con base en los resultados obtenidos se procedió a las correcciones necesarias.

Para el inicio de la aplicación de encuestas fue necesario hacer un diseño de muestra, el cual se basó en una población determinada a partir de datos del número de personas que asisten al caso de estudio en el período a analizar.

La muestra fue diseñada, con una confiabilidad del 95\% y precisión de los estimadores del 5\%. Se estimaron 380 observaciones, pero debido a la aceptación del estudio entre los encuestados se llegó a un total de 822 aplicaciones.

El proceso de selección de sujetos fue de tipo determinístico. Los sujetos eran hombres y mujeres entre 12 y 75 años de edad, no se incluyeron individuos con condiciones biológicas irregulares como enfermedades crónicas, embarazo, período de lactancia ó período menstrual. Algunas imágenes de aplicación de encuestas y equipo se presentan en la Figura 1.

Las encuestas se realizaron en los horarios de operación del parque $(6 \mathrm{~h} 00$ a $22 \mathrm{~h} 00$ de lunes a domingo), de tal forma que se cubrieron todos los períodos de uso diario en proporción al nivel de asistencia al lugar. Lo anterior sirvió para evitar que se acumularan encuestas levantadas en un horario solamente.

Previo al proceso de captura, se llevó a cabo una clasificación de las encuestas por nivel de actividad. Además de una segunda revisión para ver posibles errores en los datos capturados. Paralelo a lo anterior se diseñó una hoja de cálculo en el programa Excel, debido a su capacidad para hacer análisis de datos, además de su compatibilidad con programas especializados sobre análisis estadístico. El formato elaborado fue con base en el estudio desarrollado por GómezAzpeitia et al. (2007). La base de datos que contiene la información generada en las encuestas incluye los mismos grupos, indicadores y variables del cuestionario, así como otras variables de apoyo que fueron necesarias para el estudio.

\section{Medias por intervalo de sensación térmica}

El análisis de correlación de datos entre la sensación térmica percibida y la temperatura de bulbo seco, se llevó a cabo con el método de Medias por Intervalo de Sensación Térmica (MIST) (Ver Figura 2), propuesto por GómezAzpeitia et al. (2007), el cual fue desarrollado con base en la propuesta de Nicol (1993) para los climas "asimétricos"; que consiste en utilizar la estadística descriptiva en la determinación de un valor neutral de temperatura, que se considera la de confort térmico.

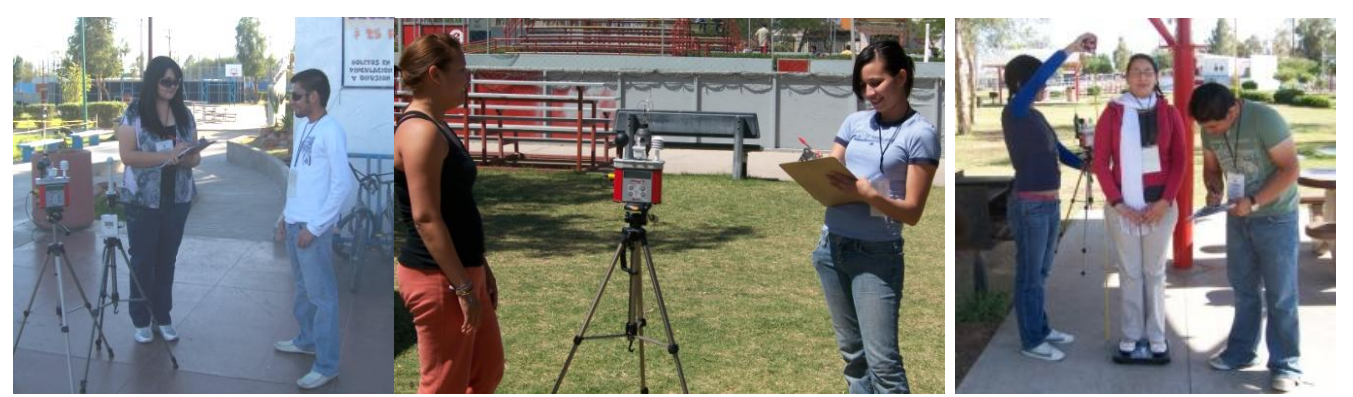

Figura 1 - Fotos de la aplicación de encuestas y monitor de estrés térmico 


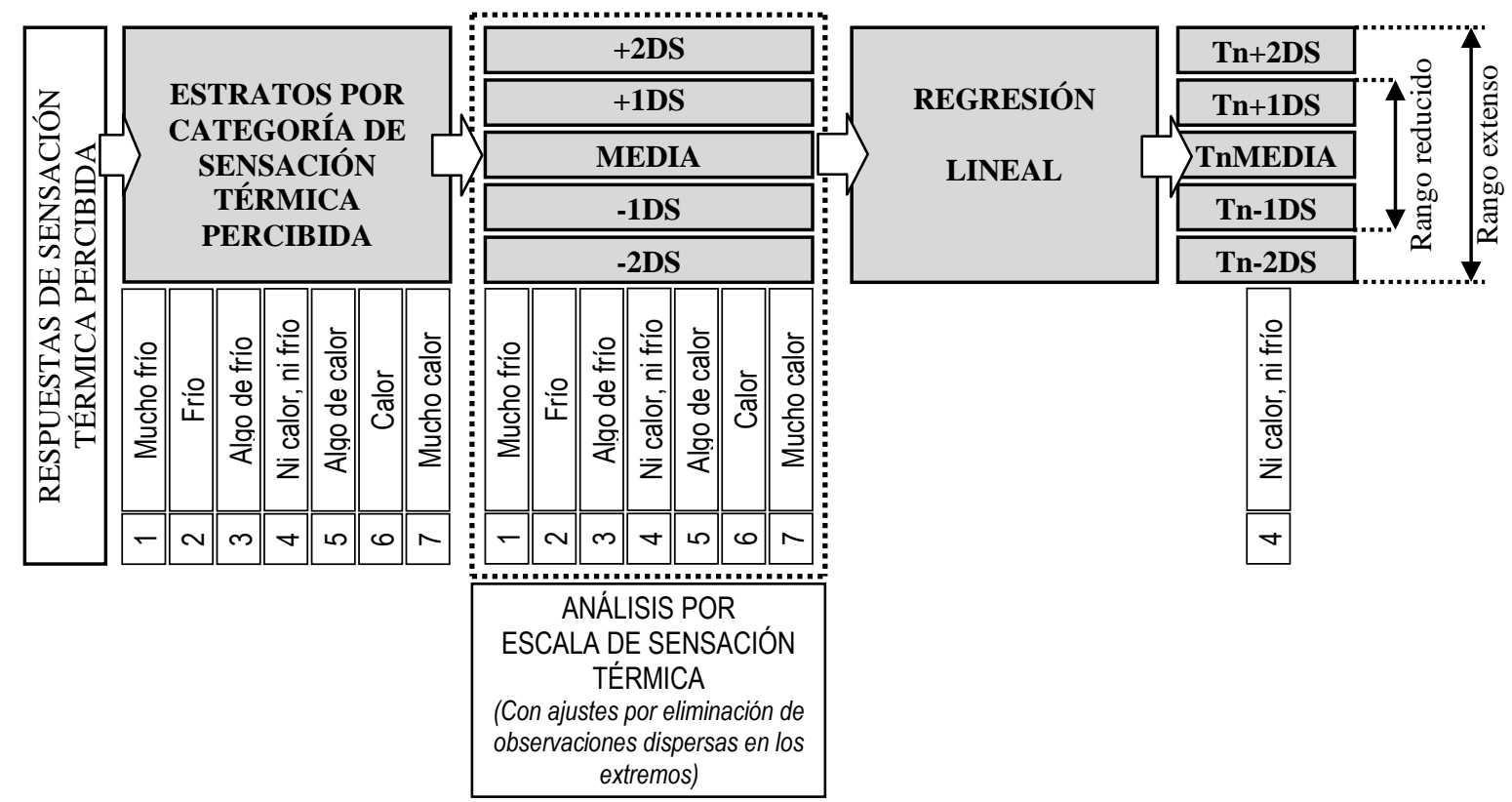

Figura 2 - Estimación de temperatura neutra (Tn) y rangos de confort térmico con el método de Medias por Intervalo de Sensación Térmica

El desarrollo del método MIST se fundamenta en el enfoque de adaptación del confort térmico. Dicho enfoque implica la interacción de variables físicas y biológicas (clima, metabolismo, aislamiento térmico) a la vez que psicológicas (adaptación, experiencia, expectativa, tolerancia, deseo). Por tal motivo la sensación de confort varía de individuo a individuo, de localidad a localidad, e incluso de estrato sociocultural a otro (BRAGER; DE DEAR, 2003).

En este sentido, la variabilidad regional y estacional de la magnitud del rango de confort es más congruente con el enfoque adaptativo que la hipótesis convencional de aplicar una amplitud fija a una temperatura neutra, aunque ésta sea variable. De acuerdo al enfoque de adaptación ambos parámetros debían ser variables.

La diferencia fundamental con el método convencional es que antes de obtener la línea de regresión que caracteriza a la muestra estudiada, se determinen grupos o estratos de la misma para calcular el valor promedio y la desviación estándar de cada uno de ellos. Así, la regresión no se hace con todos los pares de datos de la muestra, sino sólo con los valores medios y los rangos se establecen mediante la adición y sustracción de una o más veces la desviación estándar (DS) de la muestra.

El objetivo de este procedimiento es determinar el valor medio de temperatura de todas las repuestas de cada clase. De esa forma se calcula el valor de la temperatura promedio de los sujetos que dijeron sentirse en confort, pero también de quienes expresaron sentir calor o frío en cada una de las categorías previamente establecidas. En el caso de distribuciones demasiado asimétricas, es decir que la media se carga demasiado a uno de los extremos del grupo de datos y no refleja el punto medio de la muestra, se recomienda utilizar la mediana y por lo tanto la desviación estándar a la mediana.

La desviación estándar se emplea como una medida de la dispersión de las respuestas, y por lo tanto sirve para determinar los estratos en que se pueden ordenar éstas. Se estima que para datos normalmente distribuidos, el rango de $\pm 1 \mathrm{DS}$ incluye al $68 \%$ de las respuestas y \pm 2 DS incluye al $95 \%$. Para datos no distribuidos normalmente este porcentaje puede variar, por lo que se recomienda obtener el mayor número de respuestas en los estudios de campo para lograr una distribución lo más cercana a la "normal".

Para este estudio, se procesaron por separado los datos colectados en el trabajo de campo de acuerdo a cada una de las siete categorías de respuesta de confort según la ISO 10551 (ISO, 1995) que son las presentadas en la Figura 2. Se determinaron para cada una de ellas los valores promedio y desviación estándar de las temperaturas registradas para cada respuesta colectada. Cuando el número de respuestas de determinado grupo no era suficiente para obtener resultados confiables, se omitió el procedimiento y se eliminó la categoría.

Una vez que se obtuvieron estos datos se establecieron los rangos de distribución para cada 
categoría de respuesta. Se hizo a partir del valor medio de temperatura correspondiente (Media) y la adición de $\pm 1 \mathrm{DS}$. Este primer rango incluye teóricamente $68 \%$ de las personas que expresaron tener una misma sensación térmica. Se repite el procedimiento y se adiciona $\pm 2 \mathrm{DS}$ a la Media, con lo que teóricamente se incluye $95 \%$ de la población que emitió un mismo voto de sensación térmica.

Posteriormente se llevó a cabo un procedimiento de "ajuste" donde las observaciones ubicadas en los extremos de cada categoría de sensación térmica percibida que no tuvieron un comportamiento dentro de la lógica de la mayoría de las observaciones, fueron eliminadas, con lo que el valor de $\mathrm{R}^{2}$ fue más consistente.

Por último se realizó una regresión lineal con los valores que fueron obtenidos, a fin de determinar las rectas correspondientes a los límites extremos de los rangos definidos por TnMedia $\pm 2 \mathrm{DS}$, y a los límites cercanos definidos por TnMedia $\pm 1 \mathrm{DS}$. También se hizo lo mismo con los valores de TnMedia.

La intersección de cada una de las líneas de regresión con la ordenada cuatro (que representa la sensación térmica de confort, ni calor, ni frío) determinó el valor de la temperatura neutra según el método MIST, así como los valores límites de los rangos de confort térmico. Para el proceso anterior se desarrolló una hoja de cálculo en Excel. Finalmente se obtuvieron los coeficientes de determinación para los valores medios, $\pm 2 \mathrm{DS}$ y $\pm 1 \mathrm{DS}$, para efectos de validez del modelo se consideró el valor de la media.

La aplicación del método MIST, se hizo para los tres niveles de actividad (pasiva, moderada e intensa), así como una combinación de los tres niveles denominada "total de observaciones". En cada análisis, mediante el uso de tablas de datos obtenidos, se consideró una revisión de las diferencias entre la media del valor de confort térmico (escala 4, ni frío ni calor), con respecto a los valores medios de las demás sensaciones térmicas registradas, con la intención de ver la variación con respecto de una sensación térmica a otra. También se establecieron las diferencias entre las desviaciones estándar por sensación térmica percibida y las de los valores de los coeficientes de determinación para observar su variabilidad.

Con el apoyo de una gráfica, se hizo un análisis de las líneas de regresión media y $\pm 2 \mathrm{DS}$ y $\pm 1 \mathrm{DS}$, para visualizar la adaptación subjetiva de las observaciones estudiadas. También se estimó la variación entre el rango extenso y el rango reducido así como las variaciones entre los coeficientes de determinación, lo anterior para conocer la variabilidad de datos entre las sensaciones térmicas y la desviación estándar de la información recolectada.

Finalmente, se llevó a cabo un análisis comparativo sobre el valor neutral (temperatura de confort), rangos de confort térmico y coeficiente de determinación. Las comparaciones internas por cada período se hicieron en función del valor de sensación térmica para el nivel de actividad pasiva, esto con la intención de conocer los efectos de las variables estudiadas por los niveles de actividad metabólica.

Cabe mencionar que en este caso, el análisis desarrollado no hace diferenciaciones de edad o sexo en los sujetos de estudio, lo anterior con la intención de estimar modelos genéricos de fácil aplicación para la toma de decisiones en el diseño arquitectónico. Además, el efecto de la adaptación psicológica en los sujetos de estudio fue considerado de forma esencial, en función de sus respuestas y en la escala de sensaciones subjetivas utilizadas, sin llegar a hacer un análisis específico de estos aspectos.

\section{Resultados}

En este apartado se presentan los resultados obtenidos, en principio con el total de observaciones y posteriormente por cada uno de los niveles de actividad estudiados, además se incluye un análisis comparativo.

\section{Total de observaciones}

En el período estudiado se hicieron un total de 822 observaciones, las cuales incluyen, 389 con actividad pasiva, 256 con actividad moderada y 177 con actividad intensa. No se presentaron sensaciones frías en ningún nivel de actividad.

Las sensaciones térmicas para el total de observaciones, tuvieron una variación de 1,8 a 5,4 ${ }^{\circ} \mathrm{C}$, en los valores medios (Media), con respecto al valor de confort térmico (4). En lo que respecta a los valores mínimos $( \pm 1$ DS) y máximos $( \pm 2 \mathrm{DS})$ de cada nivel de sensación térmica, se obtuvo una variación máxima de $17,7^{\circ} \mathrm{C}$ (Ver Tabla 1$)$.

Al estimar la Tn y los rangos de confort térmico, para el total de observaciones, se presentaron pocos casos fuera de los límites de confort térmico. Las líneas de regresión fueron convergentes con respecto a línea de regresión media, conforme aumentó la sensación térmica de calor. La variación entre la amplitud del rango extenso y el reducido fue de $7,7 \%$. La diferencia del coeficiente de determinación $\left(\mathrm{R}^{2}\right)$ para las regresiones presentó una variación máxima de 0,0119 (Ver Figura 3). 


\section{Actividad pasiva}

En lo que respecta a la actividad pasiva se hicieron un total de 389 observaciones. Las sensaciones térmicas para actividad pasiva presentaron una variación de 1,7 a $5,2^{\circ} \mathrm{C}$ en los valores medios con respecto al valor confort térmico. En lo que respecta a los valores estimados con $\pm 1 \mathrm{DS}$ y $\pm 2 \mathrm{DS}$ por intervalo de sensació GOULART, S. V. G. n térmica, se obtuvo una variación máxima de 15,9 ${ }^{\circ} \mathrm{C}$ (Ver Tabla 2).

En la actividad pasiva, se observó que las líneas de regresión de $\pm 1 \mathrm{DS}$ y $\pm 2 \mathrm{DS}$ fueron convergentes con respecto a la línea de TnMedia. La variación entre la amplitud del rango extenso y el reducido fue de $7,3{ }^{\circ} \mathrm{C}$. La diferencia de $\mathrm{R}^{2}$ para las regresiones presentó una variación máxima de 0,0070 (Ver Figura 4).

La actividad pasiva no presentó sensaciones de frío (de 3 a 1), y además, conforme a las líneas de regresión se mostró una tendencia de mayor adaptación térmica a las sensaciones de calor (de 5 a 7). La temperatura neutral de la actividad pasiva fue simétrica con respecto a los rangos de confort térmico extenso y reducido. Lo anterior fue debido al bajo nivel de energía interna generada y su intercambio termodinámico con el ambiente térmico.

\section{Actividad moderada}

En la actividad moderada se aplicaron 256 observaciones. Las sensaciones térmicas tuvieron una variación de 0,8 a $3,1^{\circ} \mathrm{C}$ en los valores medios, con respecto al valor confort térmico. En lo que respecta a los valores estimados con $\pm 1 \mathrm{DS}$ y $\pm 2 \mathrm{DS}$ por intervalo de sensación térmica, se obtuvo una variación máxima de $17,5{ }^{\circ} \mathrm{C}$ (Ver Tabla 3).

En la actividad moderada, se observó que las líneas de regresión de $\pm 1 D S$ y $-2 D S$ fueron ligeramente convergentes con relación al valor medio, conforme aumentó el nivel de sensación térmica (mucho calor), sin embargo, en el caso de +2DS el efecto de convergencia se presentó cuando disminuyó la sensación térmica a mucho frío, lo que significó que conforme aumentó la desviación estándar fue menor el nivel de adaptación para temperaturas mayores a la Tn. La variación entre la amplitud del rango extenso y el reducido fue de $7,9^{\circ} \mathrm{C}$. La diferencia de $\mathrm{R}^{2}$ para las regresiones presentó una variación máxima de 0,6106 (Ver Figura 5).

La actividad moderada no presentó sensaciones de frío (de 3 a 1); sin embargo, conforme a las líneas de regresión se mostró una adaptación térmica similar tanto a las sensaciones de frío como a las de calor. La temperatura neutral fue ligeramente asimétrica con respecto a los rangos de confort térmico extenso y reducido. Cabe mencionar que en este nivel de actividad se presentó mayor variabilidad en la energía interna debido a las diferentes actividades que se desarrollan.

\begin{tabular}{l|c|c|c|c|c|c|c}
\hline DS & Sensación térmica & Escala & -2DS & -1DS & Media & +1DS & +2DS \\
\hline 2,8 & Mucho calor & 7 & 33,1 & 35,9 & 38,7 & 41,5 & 44,3 \\
\hline 3,1 & Calor & 6 & 31,2 & 34,3 & 37,4 & 40,5 & 43,6 \\
\hline 3,5 & Algo de calor & 5 & 28,9 & 32,3 & 35,8 & 39,2 & 42,7 \\
\hline 3,9 & Ni calor, ni frío & 4 & 26,6 & 30,5 & 34,4 & 38,3 & 42,2 \\
\hline
\end{tabular}

Tabla 1 - Media aritmética y desviación estándar para sensaciones térmicas, total de observaciones

\begin{tabular}{l|c|c|c|c|c|c|c}
\hline \multicolumn{1}{c|}{ DS } & Sensación térmica & Escala & -2DS & -1DS & Media & +1DS & +2DS \\
\hline 1,6 & Mucho Calor & 7 & 36,7 & 38,4 & 40,0 & 41,7 & 43,3 \\
\hline 2,3 & Calor & 6 & 33,7 & 36,0 & 38,3 & 40,6 & 42,8 \\
\hline 3,0 & Algo de calor & 5 & 30,6 & 33,6 & 36,6 & 39,5 & 42,5 \\
\hline 3,7 & Ni calor, ni frío & 4 & 27,4 & 31,1 & 34,8 & 38,5 & 42,2 \\
\hline
\end{tabular}

Tabla 2 - Media aritmética y desviación estándar para sensaciones térmicas, actividad pasiva 


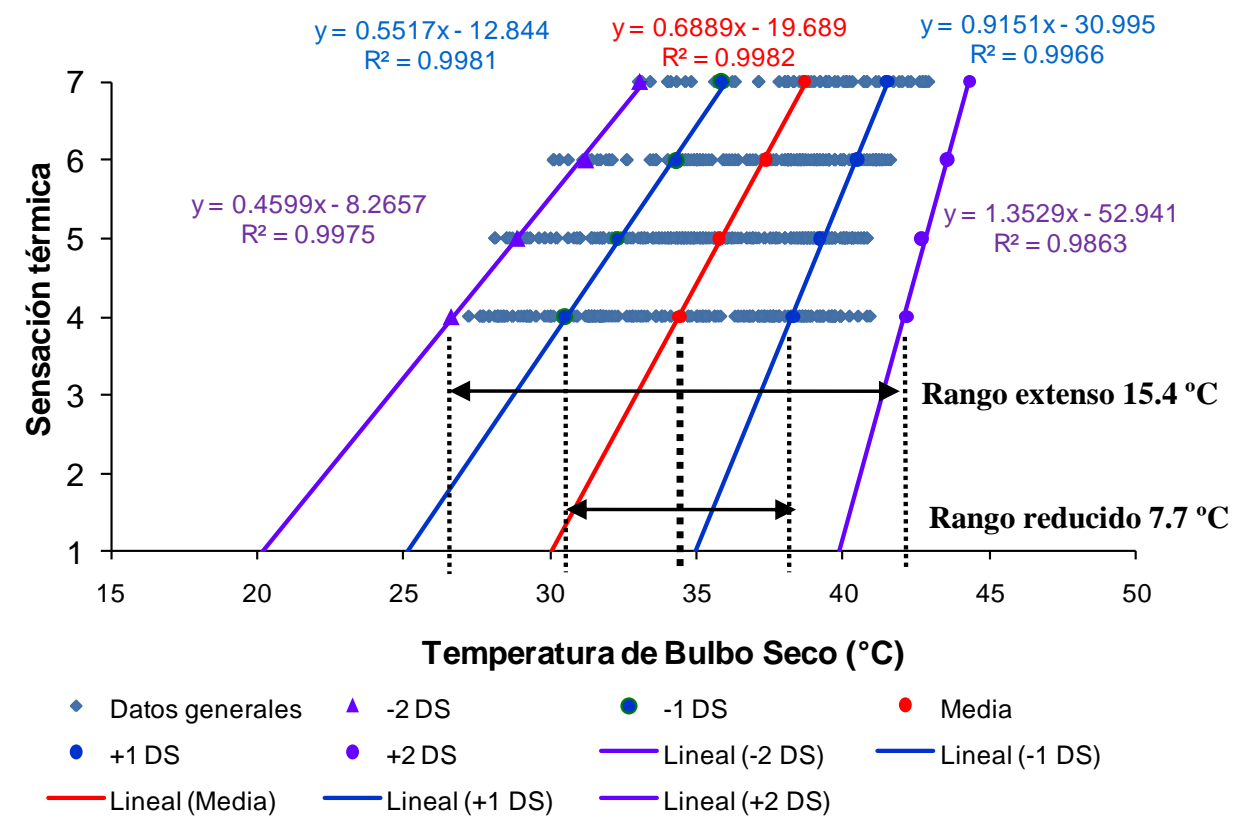

\begin{tabular}{c|c|c|c|c}
\hline Tn-2DS & Tn-1DS & TnMedia & Tn+1DS & Tn+2DS \\
\hline 26,7 & 30,5 & 34,4 & 38,2 & 42,1 \\
\hline
\end{tabular}

Figura 3 - Temperatura neutral y rangos de confort térmico para el total de observaciones

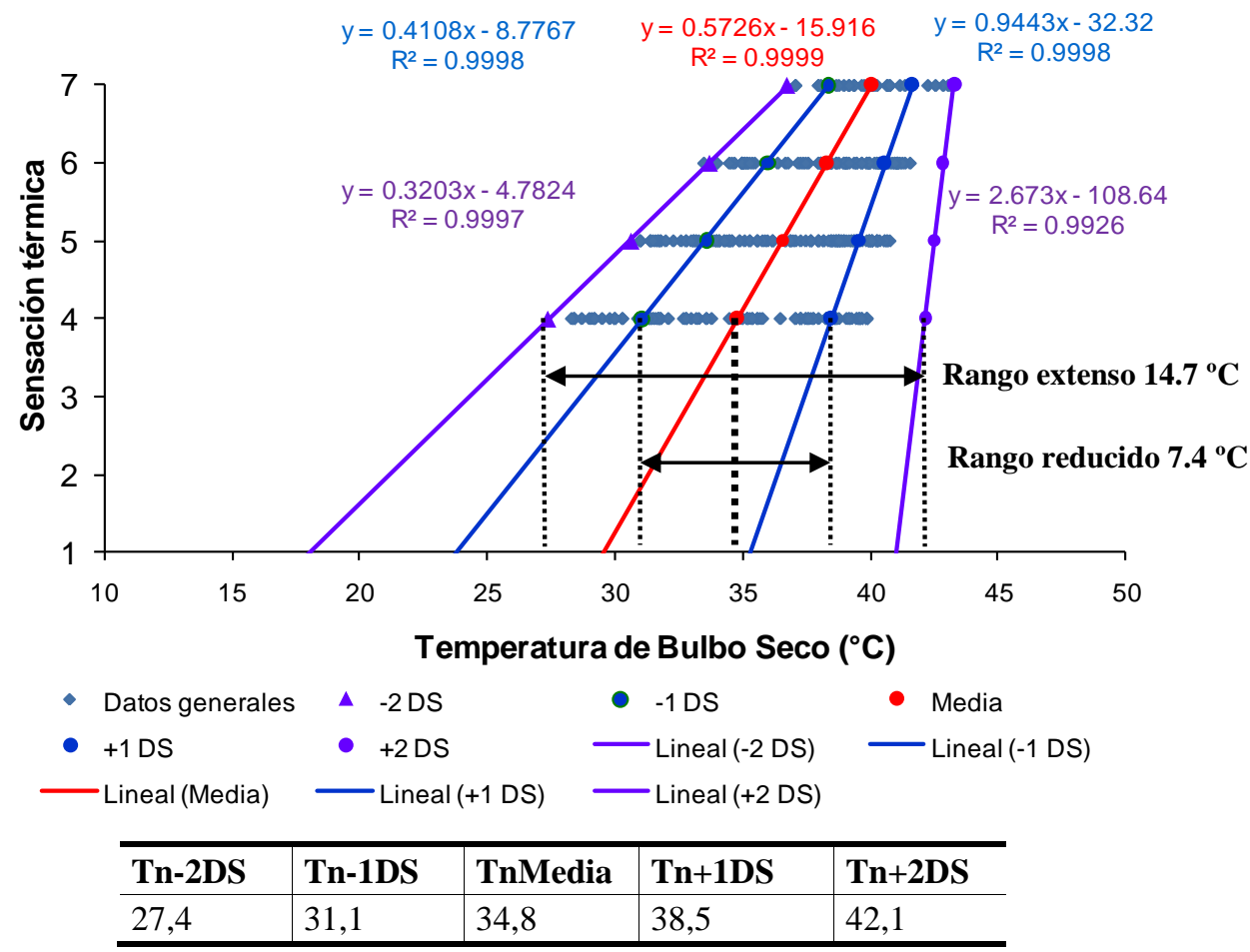

Figura 4 - Temperatura neutral y rangos de confort térmico para actividad pasiva 


\begin{tabular}{c|c|c|c|c|c|c|c}
\hline DS & Sensación térmica & Escala & -2DS & -1DS & Media & +1DS & +2DS \\
\hline 3,0 & Mucho calor & 7 & 30,1 & 33,2 & 36,2 & 39,2 & 42,2 \\
\hline 3,9 & Calor & 6 & 26,8 & 30,7 & 34,7 & 38,6 & 42,5 \\
\hline 4,4 & Algo de calor & 5 & 25,2 & 29,6 & 33,9 & 38,3 & 42,7 \\
\hline 3,9 & Ni calor, ni frío & 4 & 25,2 & 29,1 & 33,1 & 37,0 & 41,0 \\
\hline
\end{tabular}

Tabla 3 - Media aritmética y desviación estándar para sensaciones térmicas, actividad moderada

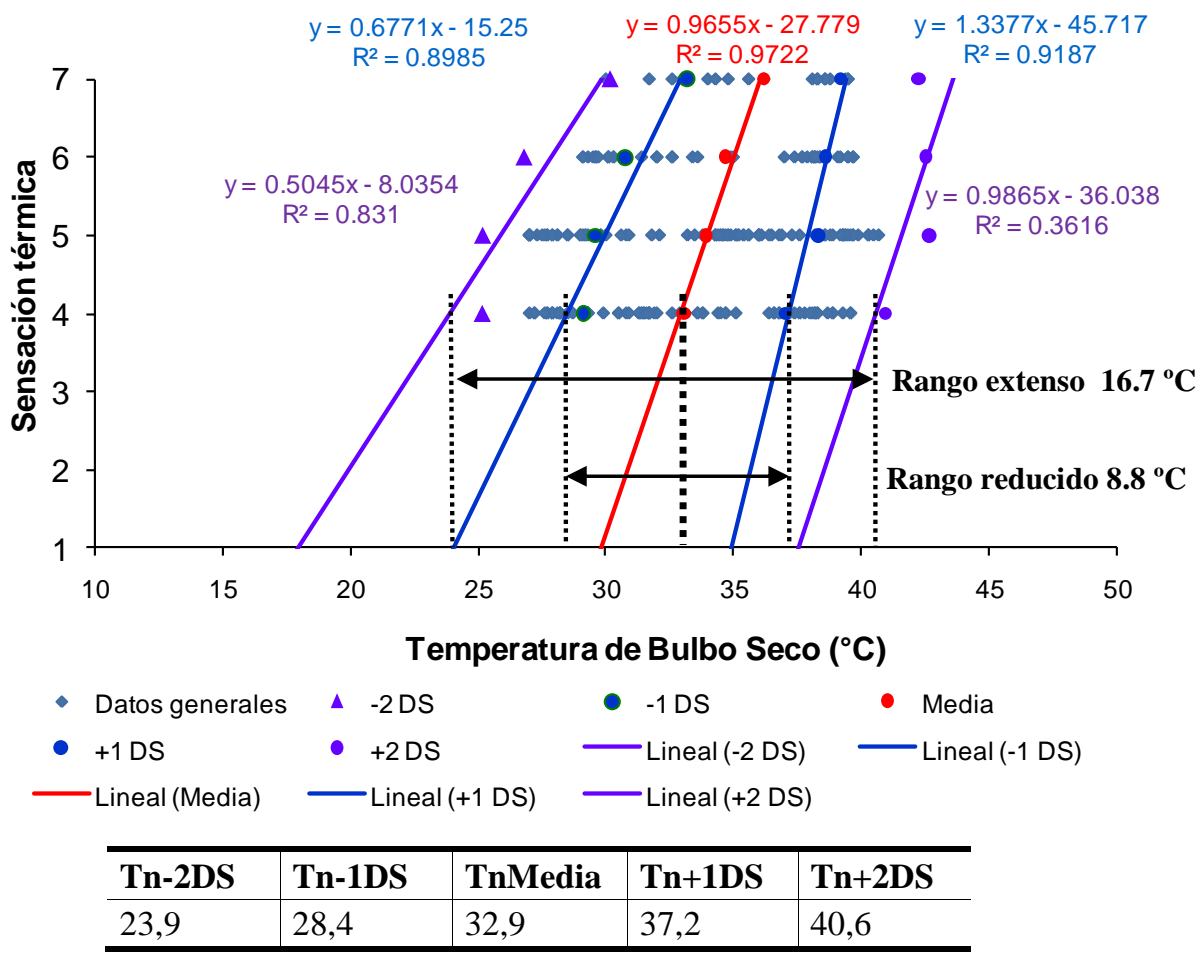

Figura 5 - Temperatura neutral y rangos de confort térmico para actividad moderada

\section{Actividad intensa}

En lo que respecta a la actividad intensa se hicieron un total de 177 observaciones. Las sensaciones térmicas tuvieron una variación de 0,6 a $3,8{ }^{\circ} \mathrm{C}$ en los valores medios con respecto al valor confort térmico. En lo que respecta a los valores estimados con \pm 1 DS y \pm 2 DS por intervalo de sensación térmica se obtuvo una variación máxima de $19.4^{\circ} \mathrm{C}$ (Ver Tabla 4).

En la actividad intensa, se observó que todas las líneas de regresión de \pm DDS y $\pm 2 D S$ fueron convergentes con respecto a la línea de regresión media conforme aumentó la sensación térmica de calor, aun cuando aparentemente son paralelas entre sí. La variación entre la amplitud del rango extenso y el reducido fue de $7,6^{\circ} \mathrm{C}$. La diferencia de $\mathrm{R}^{2}$ para las regresiones presentó una variación máxima de 0,1408 (Ver Figura 6).

La actividad intensa no presentó sensaciones de frío (de 3 a 1); sin embargo, conforme a las líneas de regresión se observó una adaptación térmica similar tanto a las sensaciones de frío como a las de calor. La temperatura neutral fue simétrica con respecto a los rangos de confort térmico extenso y reducido. Cabe mencionar que en este nivel de actividad se presentó mayor nivel de adaptación debido a la periodicidad de la práctica de actividades, así como la adaptación psicológica (expectativa y experiencia, conducta reactiva) por lo que su temperatura de confort térmico fue similar a la de los sujetos con actividad pasiva. 


\begin{tabular}{c|c|c|c|c|c|c|c}
\hline DS & Sensación térmica & Escala & -2DS & -1DS & Media & +1DS & +2DS \\
\hline 3,8 & Mucho calor & 7 & 31,4 & 35,2 & 39,0 & 42,8 & 46,5 \\
\hline 3,2 & Calor & 6 & 31,8 & 35,0 & 38,2 & 41,4 & 44,5 \\
\hline 3,6 & Algo de calor & 5 & 28,6 & 32,2 & 35,8 & 39,4 & 43,0 \\
\hline 4,1 & Ni calor, ni frío & 4 & 27,1 & 31,2 & 35,2 & 39,3 & 43,4 \\
\hline
\end{tabular}

Tabla 4 - Media aritmética y desviación estándar para sensaciones térmicas, actividad intensa

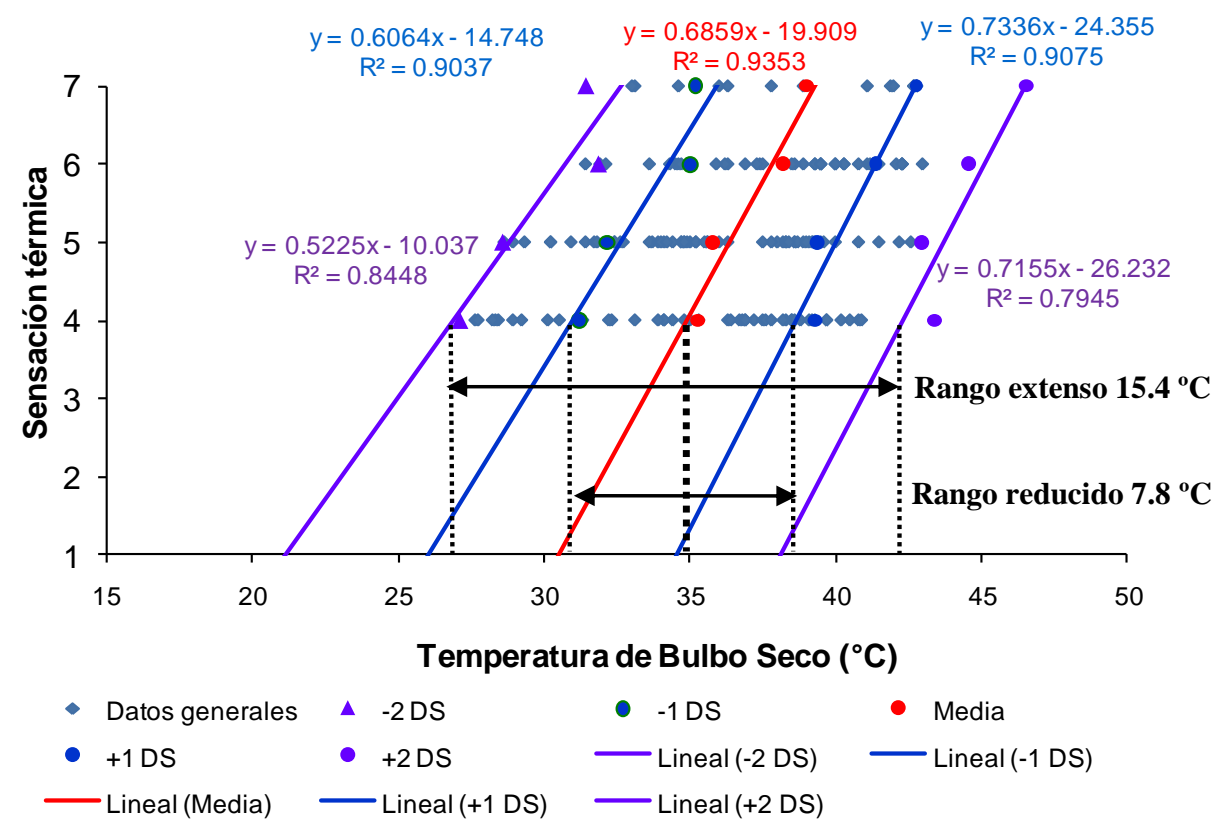

\begin{tabular}{l|l|l|l|l}
\hline Tn-2DS & Tn-1DS & TnMedia & Tn+1DS & Tn+2DS \\
\hline 26,9 & 30,9 & 34,9 & 38,7 & 42,3 \\
\hline
\end{tabular}

Figura 6 - Temperatura neutral y rangos de confort térmico para actividad intensa

\section{Análisis comparativo}

En este apartado se hace un análisis comparativo de resultados por período y nivel de actividad. Los aspectos a estudiar fueron el valor neutral (Tn: temperatura de confort), rangos de confort térmico y coeficiente de determinación.

El valor neutral para actividad pasiva fue de 34,8 ${ }^{\circ} \mathrm{C}$, mientras que para total de observaciones el valor fue $1 \%$ menor que la pasiva, la actividad moderada fue $5 \%$ menor que la pasiva y la intensa $1 \%$ mayor que la pasiva. Se observó que no hay una variación directamente proporcional entre el nivel de actividad y el aumento o reducción de los valores de Tn. Lo antes descrito se presenta en la Tabla 5 .

El rango extenso, tuvo un valor en la actividad pasiva de $14,7{ }^{\circ} \mathrm{C}$, mientras que para el total de observaciones el valor fue $5 \%$ mayor que la pasiva y en la actividad moderada fue $14 \%$ mayor, en el caso de la actividad intensa fue 5\% mayor que la pasiva (Ver Tabla 5). Para los niveles de actividad de pasivo a intenso los valores de rangos extensos estuvieron entre 23,9 y $42,3{ }^{\circ} \mathrm{C}$, variación aceptable sí se considera que el estudio se realizó en horarios de 6 h00 a 21 h30 con mínimas de $18{ }^{\circ} \mathrm{C}$ y máximas de $48{ }^{\circ} \mathrm{C}$. El rango más amplio se presentó en sujetos con actividad moderada, lo anterior se debe a la mayor variabilidad en la energía interna debido a las diferentes actividades que se desarrollan en este nivel de actividad.

El coeficiente de determinación $\left(\mathrm{R}^{2}\right)$, tuvo un valor para la actividad pasiva de 0,9999 , mientras que para total de observaciones el valor fue $1,7 \times 10^{-3} \%$ menor que la pasiva y en la actividad moderada fue $2 \%$ menor, en caso de la actividad intensa fue $6 \%$ menor que la pasiva (Ver Tabla 5). 


\begin{tabular}{l|c|c|c|c}
\hline Característica & $\begin{array}{c}\text { Total de } \\
\text { observaciones } \\
\text { (Figura 3) }\end{array}$ & $\begin{array}{c}\text { Actividad } \\
\text { pasiva } \\
\text { (Figura 4) }\end{array}$ & $\begin{array}{c}\text { Actividad } \\
\text { moderada } \\
\text { (Figura 5) }\end{array}$ & $\begin{array}{c}\text { Actividad } \\
\text { intensa } \\
\text { (Figura 6) }\end{array}$ \\
\hline Tn+2DS & 42,2 & 42,2 & 41,0 & 43,4 \\
\hline Tn+1DS & 38,3 & 38,5 & 37,0 & 39,3 \\
\hline TnMedia & 34,4 & 34,8 & 33,1 & 35,2 \\
\hline Tn-1DS & 30,5 & 31,1 & 29,1 & 31,2 \\
\hline Tn-2DS & 26,6 & 27,4 & 25,2 & 27,1 \\
\hline Rango extenso & 15,4 & 14,7 & 16,7 & 15,4 \\
\hline Rango reducido & 7,7 & 7,4 & 8,8 & 7,8 \\
\hline $\mathrm{R}^{2}$ (LRM) & 0,9982 & 0.9999 & 0,9722 & 0,9353 \\
\hline
\end{tabular}

Tn: Temperatura neutral, DS: Desviación estándar, $\mathrm{R}^{2}$ (LRM): Coeficiente de determinación de línea de regresión media.

Tabla 5 - Comparación de temperatura neutral y rangos de confort térmico para total de observaciones, actividad pasiva, moderada e intensa

Cabe mencionar que el coeficiente de determinación para actividad pasiva fue el mayor en el estudio realizado, sin embargo, en los otros niveles de actividad los valores fueron consistentes y significativos.

\section{Conclusiones}

Con base en los resultados obtenidos se establecen las conclusiones siguientes:

El período estudiado presenta un comportamiento de clima asimétrico, debido a que por condiciones extremas de calor, no se observan sensaciones frías, lo anterior demuestra la teoría de adaptación al período cálido.

A mayor convergencia de las líneas de regresión, sobre la sensación térmica de "ni calor, ni frío" mejor adaptación térmica, en otras palabras a menor varianza sobre la temperatura de confort térmico, menor variación en la sensación térmica de la muestra estudiada.

Cuando las líneas de regresión tienden a ser paralelas (visualmente) entre sí, conforme la sensación térmica percibida se hace fría o caliente, significa que la adaptación térmica es similar hacia el calor o el frío, ya que la amplitud de rangos se mantiene en cada sensación térmica percibida, en otras palabras, sí la pendiente de la recta es similar para $\pm 1 \mathrm{DS}$ y $\pm 2 \mathrm{DS}$, el nivel de adaptación para cada sensación térmica percibida es similar también.

Se observó una variación de la temperatura de confort térmico entre un nivel de actividad y otro. Esto se debe a la diferencia de los niveles de arropamiento, periodicidad de la actividad y adaptación térmica y psicológica de los sujetos.

La variabilidad en la simetría y asimetría de las líneas de tendencia en los tres niveles de actividad, se debe a la diversidad de niveles de aclimatación y conductas reactiva e interactiva de los sujetos.
Los valores de TnMedia obtenidos presentan similitud para el estudio realizado por GómezAzpeitia et al. (2009), en interiores en la misma ciudad de estudio.

La temperatura neutral presenta variaciones entre los niveles de actividad, pudiera suponerse que conforme aumenta el nivel de actividad se reduce la temperatura de confort térmico, sin embargo, en el caso de la actividad intensa los sujetos están mejor adaptados, que los de actividad moderada, debido a la periodicidad de la práctica de actividades, esto coincide con Rhoades y Tanner (1997), Humphreys y Nicol (2002), así como la adaptación psicológica (expectativa y experiencia, conducta reactiva) por lo que su temperatura de confort térmico es similar a la de los sujetos con actividad pasiva.

La mayor variación en las sensaciones térmicas se presenta en la actividad moderada, debido a las distintas actividades y rangos de metabolismo global de los mismos.

En el caso de los rangos de confort térmico, el rango extenso más amplio se da en la actividad de nivel moderado, con similitud para actividades pasivas. Lo anterior se debe a que las actividades en este nivel, presentan condiciones de variabilidad amplias en lo que respecta a su demanda metabólica ya que están en rangos que en ocasiones son similares a actividades pasivas o intensas; lo que ocasiona diferencias significativas en el nivel de adaptación de los sujetos.

El rango reducido presenta su valor mayor en la actividad pasiva e intensa. Lo anterior se debe a que en período cálido se manifiesta una amplitud en los valores fuera de confort térmico en actividades pasivas e intensas.

Los cambios de adaptación mayores ocurren en la actividad intensa, mientras que los menos significativos se dan en la actividad pasiva, debido 
a la condición física y expectativa y experiencia de las personas.

El estudio sirve como apoyo en la toma de decisiones de diseño de espacios exteriores en clima desértico, ya que permite estimar las condiciones de confort térmico de los usuarios, en tres distintos niveles de actividad para condiciones extremas de calor.

\section{Referências}

ASHIHARA, Y. El diseño de Espacios

Exteriores. Barcelona: Gustavo Gili, 1982.

AULICIEMS, A. Global Differences in Indoor Thermal Requirements. In: AUSTRALIAN \& NEW ZEALAND ASSOCIATION FOR THE ADVANCEMENT OF SCIENCE CONFERENCE, 1998, Brisbane. Proceedings... Brisbane: ANZAAS, 1998. p. 98-105.

BRAGER, G.; DE DEAR, R. Historical and Cultural Influences on Comfort Expectations. In: COLE, R.; LORCH, R. (Ed.). Buildings, Culture and Environment: informing local and global practices. Blackwell: London, 2003. p. 177-201.

DE DEAR, R.; BRAGER, G.; COOPER, D. Developing an Adaptive Model of Thermal Comfort and Preferentes: final report on RP-884. Atlanta: ASHRAE: Macquarie Research, 1998.

FANGER, P. O. Thermal Environment-Human Requirements. Environmentalist, Hampshire, v. 6, n. 4, p. 275-278, 1986.

GIVONI, B. et al. Outdoor Comfort Research Issues. Energy and Buildings, Lausanne, n. 35, p. 77-86, 2003.

GÓMEZ-AZPEITIA, G. et al. Comfort Temperatures inside Low-Cost Housings of Six Warm Climate Cities in Mexico. In: CONFERENCE ON PASSIVE AND LOW ENERGY ARCHITECTURE, 26., 2009, Quebec. Proceedings... Quebec: PLEA, 2009. p. 122-128.

GÓMEZ-AZPEITIA, G. et al. Monitoreo de Condiciones de Confort Térmico: reporte técnico. Colima: CONAFOVI, 2007.

HÖPPE, P. Different aspects of assessing indoor and outdoor thermal comfort. Energy and Buildings, Lausanne, n. 34, p. 661-665, 2002.

HUMPHREYS, M.; NICOL, F. The Validity of ISO-PMV for Predicting Comfort Votes in EveryDay Thermal Environments. Energy and Buildings, Lausanne, n. 34, p. 667-684, 2002.

HUMPHREYS, M.; NICOL, F. Understanding the Adaptive Approach to Thermal Comfort.
ASHRAE Technical Bulletin, Atlanta, v. 14, n. 1, p. 27-49, 1998 .

INTERNATIONAL ORGANIZATION FOR STANDARDIZATION (ISO). ISO 7726:1998(E): ergonomics of the thermal environment: instruments for measuring physical quantities. Ginebra, 1998.

INTERNATIONAL ORGANIZATION FOR STANDARDIZATION (ISO). ISO 7730:2005(E): ergonomics of the thermal environment: analytical determination and interpretation of thermal comfort using calculation of the PMV and PPD indices and local thermal comfort criteria. Ginebra, 2005.

\section{INTERNATIONAL ORGANIZATION FOR}

STANDARDIZATION (ISO). ISO

10551:1995(E): ergonomics of thermal environment: assessment of the influence of the thermal environment using subjective judgment scales. Ginebra, 1995.

LUNA, A. et al. Aire Acondicionado Solar para Conjunto de Viviendas en Mexicali, B.C.

Información Tecnológica, México, v. 19, n. 1, p. 45-56, 2008

MONDELO, P. et al. Ergonomía 2: confort y estrés térmico. 3. ed. Barcelona: Universidad Politécnica Catalunya, 2001.

NICOL, F. Thermal Comfort: a handbook for field studies toward an adaptive model. London: University of East London, 1993.

NIKOLOPOULOU, M. Designing open space in the urban environment: a bioclimatic approach. Attiki: Center for Renewable Energy Sources, 2004.

NIKOLOPOULOU, M.; STEEMERS, K. Thermal Comfort and Psychological Adaptation as a Guide for Designing Urban Spaces. Energy and Buildings, Lausanne, n. 35, p. 95-101, 2003.

PICKUP, J.; DE DEAR, R. An Outdoor Thermal Comfort Index (OUT_SET*): part I: the model and its assumptions. In: DE DEAR, R. et al. (Ed.).

Biometeorology and Urban Climatology at the Turn of the Millennium: WCASP 50: WMO/TD N. 1026. Geneva: WMO, 2000. p. 279-283.

POTTER, J. C.; DE DEAR, R. Field Study to Calibrate an Outdoor Thermal Comfort Index. In: DE DEAR, R. et al. (Ed.). Biometeorology and Urban Climatology at the Turn of the Millennium: WCASP 50: WMO/TD N. 1026. Geneva: WMO, 2000. p. 315-320.

RHOADES, R.; TANNER, G. Fisiología Médica. Barcelona: Masson, 1997. 


\section{Agradecimientos}

Al Personal de Apoyo en el trabajo de campo, captura y procesamiento de información. Ing. Emmanuelle Maria Vasconcelos Matos y Dr. Augusto Arredondo por su apoyo durante el desarrollo de este documento. A autoridades y personal del Centro Recreativo Juventud 2000, por todas las facilidades otorgadas. Al proyecto
"Confort térmico y ahorro de energía en la vivienda económica en México: regiones de clima cálido seco y húmedo", CONAFOVI 2004-01-20 y al CONACYT- México por el apoyo económico. A Universidad Autónoma de Baja California y Universidad de Colima a través de sus Facultades de Arquitectura y Diseño, por el apoyo brindado para la realización de este trabajo. 